\title{
Which way to cooperate
}

\author{
Todd R. Kaplan \\ University of Exeter \\ Bradley J. Ruffle \\ Ben-Gurion University
}

June 2007

\begin{abstract}
Cooperation in real-world dilemmas takes many forms. We introduce a class of two-player games that permits two distinct ways to cooperate in the repeated game. One way to cooperate is to play cutoff strategies, which rely solely on a player's private value to defection. The second cooperative strategy is to take turns, which relies on publicly available information. Our initial experiments reveal that almost all cooperators adopt cutoff strategies. However, follow-up experiments in which the distribution of values to defection are made more similar show that all cooperators now take turns. Our results offer insight into what form a cooperative norm will take: for mundane tasks or where individuals otherwise have similar payoffs, taking turns is likely; for difficult tasks that differentiate individuals by skill or by preferences, cutoff cooperation will emerge.
\end{abstract}

Keywords: experimental economics, cooperation, incomplete information, alternating, cutoff strategies, random payoffs.

JEL classification nos.: C90, Z13.

Contact Information: Ruffle: Department of Economics, Ben-Gurion University, Beer Sheva 84105, Israel, e-mail: bradley@bgu.ac.il, tel.: +972-8-6472308, fax: (917) 591-8151; Kaplan: Department of Economics, University of Exeter, Exeter, UK, e-mail: T.R.Kaplan@exeter.ac.uk, tel.: +44-1392-263237, fax: (530) 8716103.

Acknowledgements: We thank Douglas Bernheim, Valin Dittrich, Dirk Engelmann, Jim Engle-Warnick, Naomi Feldman, Guillaume Fréchette, Andrew Muller, Jim Myton, Ariel Rubinstein, Tim Salmon, and Mike Stutzer for valuable comments as well as seminar participants at Bar-Ilan, Ben-Gurion, East Anglia, Emory, Florida State, Haifa, Hebrew University-Jerusalem, McMaster, Northern Illinois, Osaka, Ryerson, Technion, Tel Aviv, Tokyo and Waseda Universities, Harvard Business School, MIT Sloan School of Management, the Universities of Birmingham and Exeter, 2005 and 2006 ESA European meetings, 2005 SAET meetings, 2005 Zeuthen Workshop, 2006 DmEp Conference and the 2006 Israel Economic Association meetings. Tzvi Kerpzseld, Kadia Kohelet, Shy Maman, Tim Miller, Eran Ozery, Gad Rotman, Asaf Shaul, Tal Shavit, Ron Stern and Yael Uzen offered valuable research assistance. Urs Fischbacher provided us with his z-Tree software. Kaplan thanks the Economic and Social Research Council (grant no. RES-000-22-0370) and Ruffle thanks the Israel Science Foundation (grant no. 776/03) for generous financial support. We gratefully acknowledge support from Ben-Gurion University and the University of Exeter. 


\section{Introduction}

Many real-world cooperation dilemmas permit multiple modes of cooperation. Cab drivers at a taxi stand are assigned to passengers according to an agreed upon order, whereas roaming cabbies divvy up the dispatcher's calls on a case-by-base basis, mostly by proximity to the passenger's location. Siblings can divide household chores in a given week on a volunteer basis or by taking turns. Firms that face one another in multiple markets can collude by alternately competing and acquiescing in markets or by staying out of less profitable markets.

Why are some cooperative dilemmas resolved by taking turns or alternating, while others are best dealt with by conditioning one's cooperative behavior on the value to defection, that is, cutoff cooperation? In this paper, we aim to understand the features of cooperation dilemmas that are conducive to alternating versus cutoff cooperation. For the purpose of studying the way in which players cooperate, we introduce a class of two-player games that permits these two distinct modes of cooperation in the repeated game. To illustrate, consider the following game parameterization: each player privately receives a randomly drawn integer between 1 and 5 inclusive, each with equal probability. Each player then decides between one of two actions: enter or exit. By exiting a player receives zero. By entering, he receives his number if his opponent exits and one-third of his number if his opponent also enters.

The first-best outcome of this game entails the high-value player entering and the other player staying out. This can be achieved only when players know or learn each others' values for the action before making a decision. Two strangers who reach the airport check-in counter at the same time with tickets in hand can achieve the highest social surplus by having the person whose flight does not leave for another four hours allow the hurried passenger to go ahead. Pregnant women with children in arms are invited to bypass lengthy lineups.

In our game in which values are private and cannot be communicated or signaled, the firstbest outcome is no longer feasible. Instead, when the same pair of players interacts repeatedly, cutoff cooperation and alternation are two cooperative norms that avoid the inefficient stage- 
game dominant strategy of always entering. Cutoff cooperation entails entering when the value to doing so exceeds some threshold and not entering otherwise. Cutoff strategies thus condition on players' private information. Firms might implicitly collude by staying out of relatively high-cost or low-demand markets with the expectation that rival firms will reciprocate. Auction participants might bid only when the object is sufficiently valuable so as not to inflate the winning bid unnecessarily or receive the object when another bidder values it more.

Unlike cutoff cooperation, alternating ignores private values; rather, it makes use of publicly available information, like the time of day, the round or object number. Cooperation dilemmas in families are often resolved by alternating. Spouses take turns making important decisions; parents avoid favoring one child over another by rotating favors between them; and siblings settle scores by recalling who enjoyed the same privilege (like riding in the front seat) last time. Firms that compete with one another in multiple markets or in the same markets repeatedly, or bidders who compete for similar objects auctioned off sequentially can cooperate by taking turns capturing the market, instead of pricing or bidding aggressively in each market. Zillante (2005) presents evidence that the four baseball-card manufacturers alternate the timing with which they introduce new product lines in order to reduce intra-period competition. ${ }^{1}$

To address the questions of whether cooperation will emerge and what form it will take, we begin with the above game parameterization, which yields very similar joint expected payoffs for the socially optimal cutoff strategy and the alternating strategy. We conduct this game for 80 rounds under two private-information treatments that differ according to the point in time at which a player learns his opponent's number (at the end of the round or not at all). We find that the socially optimal symmetric cutoff strategy whereby a player enters on the numbers 3,4 and 5 , and exits otherwise is subjects' modal choice in both treatments. Revealing opponents' numbers at the end of the round is particularly conducive to cutoff strategies since entry on low values is observable and punishable. But even when the opponents' numbers are not revealed, cooperative

\footnotetext{
1 Zillante (2005) discusses other known examples, such as the motion-picture and electrical switchgear industries, in which new-product-release dates have been staggered to blunt head-on competition.
} 
cutoff strategies continue to be employed by over $70 \%$ of subjects even though play according to these strategies cannot be observed. Few subjects adopt alternation in either treatment.

In an effort to understand why so few subjects alternate, despite the strategy's prevalence in real-world cooperation dilemmas, we designed an additional pair of treatments in which we added a constant of 100 to all of the entry values. This change makes the entry values relatively similar, thereby reducing the importance of players' private information, while leaving unchanged the inherent difficulty coordinating on alternation. The results from these follow-up experiments are discussed in section 7 .

In the next section, we develop the theoretical framework for this class of two-player games and through numerical optimization arrive at the above parameterization for our experiments. We contrast our game with familiar cooperation and coordination games in section 3 . In section 4, we detail our experimental design and procedures. Section 5 presents theoretical results on cooperation for the infinitely repeated game, which yield testable experimental hypotheses. Section 6 presents the results and analysis. We attempt to understand differences in cooperative behavior between treatments and especially the paucity of alternating in these experiments. Section 7 reports the results of additional treatments designed to determine whether the inherent coordination problem with alternating may be overcome. Section 8 concludes with insights into when to expect alternation versus cutoff strategies in real-world cooperation dilemmas.

\section{Theoretical Framework}

In this section, we introduce a class of two-player games. We derive the theoretical properties of these games, which may be of some independent interest since these games are new. Moreover, the theoretical and comparative-statics results will help guide our choice of game parameterization for the subsequent experiments. While our experimental test involves a repeated game, we begin with an analysis of the one-shot game to highlight some of the differences between this and familiar cooperation games. All proofs appear in Appendix A. These results serve as a basis for 
further analysis of the repeated game (in section 5).

\section{$2.1 \quad$ Environment}

We propose a two-player game with the following general structure. Each player receives a randomly drawn number between $\underline{v}$ and $\bar{v}$ inclusive where the probability of receiving a number $x$ is $\pi_{x}$ (where $\pi_{x}>0$ and $\sum_{x \in\{\underline{v}, \ldots, \bar{v}\}} \pi_{x}=1$ ) and faces a binary decision $A=\{$ enter, exit $\}$. By exiting a player receives zero. By entering he receives his number if the other player exits or some function $f(x, y)$ increasing in his number, $x$, and possibly also a function of the other player's number, $y$, if both enter. We assume that $f(x, y)$ is strictly less than his number $x$; hence entry imposes a negative externality on the other player. We also assume that if it is profitable for a player to enter alone $(x>0)$, then it is also profitable for him to enter when his opponent enters $(f>0$ for $x>0)$. For the purposes of this paper, we consider games in which a player's number is his private information.

\subsection{Solutions}

There are noncooperative and cooperative solutions to this game. If each player is concerned about maximizing only his own payoff, then we can solve for the Bayes-Nash equilibrium. This yields the dominant strategy of entry for numbers greater than zero.

The cooperative solution is given by the pair of strategies that maximizes the sum of the players' expected payoffs. Suppose the other player enters with probability $p(y)$ when his number is $y$. The joint expected payoff to entering with number $x$ is,

$$
\sum_{y \in\{\underline{v} \ldots \bar{v}\}} \pi_{y}\{x(1-p(y))+p(y)[f(x, y)+f(y, x)]\} .
$$

The joint expected payoff to staying out is $\sum_{y \in\{\underline{v}, \ldots, \bar{v}\}} \pi_{y} y p(y)$.

Lemma 1: If $f$ is increasing in both arguments, then the cooperative solution entails cutoff strategies (that is, for $\underline{v} \leq y<\bar{v}$ if $p(y)>0$, then for all $y^{\prime}>y, p\left(y^{\prime}\right)=1$ ). 
Monotonicity explains Lemma 1: if it is profitable to enter with number $x$, then it is also profitable to enter with any number greater than $x$. A pure-strategy cutoff is when there exists a $c^{*}$ such that for all $x \leq c^{*}, p(x)=0$ and for all $x>c^{*}, p(x)=1$. A mixed-strategy cutoff is when there exists an $x$ such that $0<p(x)<1$. The lemma implies that it is never optimal to mix on more than one number in the range.

These cutoff values may be non-interior and even asymmetric. If $f(x, y)+f(y, x)>\max \{x, y\}$, then the social optimum is non-interior - both enter. An extreme form of asymmetric purestrategy cutoffs involves one player entering for all numbers greater than or equal to $\underline{v}$ (i.e., always enter) and the other entering for numbers greater than $\bar{v}$ (i.e., always exit). In a repeated game, this cooperative solution can admit the form of players taking turns entering and exiting. This solution may only reasonably be expected in games in which the same pair of players interacts repeatedly. All of our experiments are such.

\subsection{Choosing a Particular Game}

From this general framework, we selected a game to test experimentally with the goal of determining the form of cooperation. To choose a particular game, we performed numerical optimization on the space of games in which players' numbers are drawn from a uniform distribution of integers between $\underline{v}$ and $\bar{v}$ inclusive. We restricted $f(x, y)$ to be of the form $x / k$ (where $k$ is an integer) to aid subjects' understanding of the game.

Our objectives were twofold: 1) to design a game for which the joint expected payoffs from alternation and the socially optimal pure-strategy symmetric cutoff strategy are very similar; 2) to maximize the difference between the joint expected payoffs from playing the optimal symmetric pure-strategy cutoff, $c^{*}$, and the second-best symmetric pure-strategy cutoff. Put another way, we want to maximize the steepness of the joint expected payoff function around the socially optimal pure-strategy cutoff. Achieving this second goal maximizes the incentive for those players wishing to cooperate to enter for numbers greater than $c^{*}$ and exit for numbers less than $c^{*}$. Deviations 
from this strategy can thus be interpreted as an intention not to cooperate optimally.

Before computing the game that maximizes these objectives, we first derive several theoretical propositions and comparative-statics results for this class of games. These results will help narrow the range of parameters from which we determine the optimal game.

Proposition 2: $\quad$ The socially optimal pure-strategy symmetric cutoff for integers drawn independently from the uniform distribution of integers from $\underline{v}$ to $\bar{v}$ and congestion parameter $k$ is given by,

$$
c^{*}=\frac{-1-2 \bar{v}+(2 \underline{v}-1) k+\sqrt{12 \bar{v}(1+\bar{v})(k-1)^{2}+(1+2 \bar{v}+k-2 \underline{v} k)^{2}}}{6(k-1)} .
$$

Although the expression for $c^{*}$ seems unintuitive, it demonstrates the uniqueness of the socially optimal cutoff and leads to some sensible comparative-statics results. First, as the congestion parameter, $k$, increases, so does the optimal symmetric cutoff for a given $\underline{v}$ and $\bar{v}$. Intuitively, as $k$ increases, it becomes increasingly costly for both players to enter; as a result, the socially optimal threshold for entry increases. Taking the limit of $c^{*}$ as $k$ tends to infinity yields,

$$
\lim _{k \rightarrow \infty} c^{*}=\frac{-1+2 \underline{v}+\sqrt{(1-2 \underline{v})^{2}+12 \bar{v}(1+\bar{v})}}{6} .
$$

Moreover, taking $k$ and $\underline{v}$ as fixed, the expression for $c^{*}$ also reveals that as $\bar{v}$ increases, so does the socially optimal cutoff.

Corollary 3: $\quad$ For integer numbers uniformly distributed on $[\underline{v}, \bar{v}], \underline{v}<\bar{v}$, and $k \geq 3$, the socially optimal cutoff always involves each player exiting on at least the integer $\underline{v}$.

The surprising aspect of Corollary 3 is that no matter how small the percentage difference between the highest and lowest integers in the range of numbers, the socially optimal cutoff always involves some measure of cooperation by exiting on at least the lowest integer, $\underline{v}$, in the range. 
Proposition 4: For $k \leq 2$, the socially optimal strategy is a cutoff strategy. In the uniform case, as $k \rightarrow \infty$, the socially optimal strategy is alternating. ${ }^{2}$

In our search for a parameterization that yields similar joint expected payoffs for the optimal cutoff and alternating strategies, Proposition 4 suggests values of $k$ greater than 2 , but not too large: we allowed $k$ to vary from 2 to 5 . Over the range of numbers, $\{\underline{v}, \ldots, \bar{v}\}$, we allowed $\bar{v}$ to be any integer greater than or equal to 3 , and fixed $\underline{v}=1 .^{3}$ This latter decision was made because if $\underline{v}$ is an integer less than 1 , then the strategy "always enter" is no longer a unique dominant strategy in the stage game.

For our experiments, we chose $(\bar{v}=5, k=3)$. Figure 1 displays the results of our search for the range of numbers $\{1, \ldots, 5\}$ and $k \in\{2,3,4,5\}$. The figure reveals that the optimal pure-strategy cutoff value, $\mathrm{c}^{*}$, equals 1.5 for $k=2$, equals 2.5 for $k=3,4$, and equals 3.5 for $k \geq 5$. We express all cutoffs as halves to denote unambiguously that the player enters on all integers greater than the cutoff and exits otherwise. The figure also shows that the steepness around $c^{*}$ is maximized for $k=3$. For $k=3$, the pair's expected payoff if each player employs the optimal cutoff, $c^{*}=2.5$, is 2.88 . For $c=3.5$, the pair's expected payoff decreases to 2.64 and to 2.61 for $c=1.5$. Table 1 indicates the pair's joint expected payoff for all pure-strategy cutoffs and alternating.

\section{[insert Figure 1 and Table 1 here]}

\footnotetext{
${ }^{2}$ When the distribution of values is not uniform, Proposition 4 does not generally hold. Take for example the values of 100 with probability $1 / 3$ and 1 with probability $2 / 3$. For large $k$, alternating yields a joint expected payoff of 34 . Entering only when one has 100 yields 100 with probability $4 / 9$ and $\epsilon$ otherwise. Hence, this optimal cutoff strategy yields a higher joint expected payoff.

${ }^{3}$ In selecting parameters, for a given $f$, we can often increase the steepness of the joint expected payoff function around the socially optimal pure-strategy cutoff by shrinking the number of integers in the range $\{\underline{v}, \ldots, \bar{v}\}$ (i.e., by lowering $\bar{v}$ in our case). However, if the optimal cutoff is in mixed strategies, this need not be true. Instead, the joint expected payoff function connecting the two pure-strategy cutoffs that straddle the optimal mixed-strategy cutoff can be rather flat. Indeed the optimal symmetric cutoffs are in mixed strategies for $(\bar{v}=3, k=4)$, $(\bar{v}=4, k=4),(\bar{v}=5, k=2)$ and $(\bar{v}=5, k=4)$. An optimal solution in mixed strategies should be avoided due to the salience of the nearby, almost optimal, pure strategies, the improbability that both subjects will solve for, and play, the optimal mixed-strategy cutoff and the added difficulty in determining whether subjects are playing mixed strategies.
} 
For our chosen parameterization, the alternating strategy earns the pair 3 units of profit in expectation, a mere 0.12 units more than than the optimal symmetric cutoff, $c^{*}=2.5$. That these two strategies perform almost equally well despite their qualitatively very different natures raises the empirical question of which one, if any, will be adopted by players. Not only is the pair's expected payoff from playing the alternating strategy (3) higher compared to the optimal cutoff strategy (2.88), the variance of the expected payoff is also lower: 2 compared to 2.42 .

\section{Related Games}

The best known and most frequently tested cooperation game, the prisoners' dilemma (PD), has a unique dominant-strategy equilibrium in the one-shot game in which both players defect; however, if both players could commit to cooperation, both would be better off. Likewise, in the public-goods game (PG) (an n-player extension of the PD), the socially optimal outcome involves all players contributing their endowments to a public good, which conflicts with the unique dominant-strategy equilibrium in which each player to a private good. Noncooperation (enter) is also the unique dominant-strategy equilibrium of our class of games. Unlike the PD and PG games, the socially optimal outcome in our game involves one person playing his dominant strategy and the other playing his dominated strategy. A second distinction of our game is that if both players choose their dominant strategies they are better off than if both play their dominated strategies.

Van de Kragt, Dawes and Orbell (1983) introduce a variation on the standard public goods game in which a minimum aggregate contribution to the public good is required for its provision. In these threshold or step-level public goods games, there is no dominant strategy since each player would prefer to contribute if and only if his contribution is pivotal in ensuring the public good's provision.

Amnon Rapoport and his coauthors have conducted various versions of a market-entry game first introduced by Kahneman (1988). In an early version, Rapoport (1995), $n$ symmetric players 
independently decide whether to enter a market with capacity $c \leq n$. Staying out yields a fixed payoff, whereas entering yields a payoff that decreases in the number of entrants and yields less than the fixed payoff from staying out in the case of excess entry. ${ }^{4}$ In subsequent versions of the market-entry game, Rapoport and coauthors have explored the effect of deciding whether to enter in one of two markets where each market's capacity changes in each period (Rapoport, Seale and Winter, 2000) and asymmetric entry costs that are held constant throughout the experiment (Rapoport, Seale and Winter, 2002). These games have large numbers of pure-strategy and mixed-strategy equilibria, all efficient and all characterized by some subset of players entering with positive probability. By contrast, our games have a unique Nash equilibrium, which is inefficient and at odds with the full-information, social optimum whereby one player enters and the other exits. Moreover, exit is a strictly dominated strategy in our game for $\underline{v}>0$ and $f>0$. Put another way, if both players enter ("excess entry"), unlike the market entry game, each entrant still earns more than if he had exited.

For a particular realization of players' numbers, the $2 \times 2$ payoff matrix in Table 2 makes precise the outcome differences between our game and others. The top row and left column are the cooperate/exit/swerve action (depending on the game in question). The bottom row and right column are the defect/enter/not swerve action. Normalizing the off-diagonal payoffs to $(1,0)$ and $(0,1)$, we denote the payoffs from the cooperative outcome as $(a, a)$ and from the defect outcome as $(b, b)$.

\section{[insert Table 2 here]}

The PD and PG games restrict $a>b>0$ and $a<1$, but $2 a>1$ in order for the cooperative outcome to be efficient. The market-entry game requires $a=0$ and $b<0$. The game of chicken can be characterized by $1>a>0>b$. It has the same asymmetric equilibria as the marketentry game and can be seen as a limiting case of it as $a$ decreases to 0 . For both games, the

\footnotetext{
${ }^{4}$ The special case in which the payoff for entering changes only in going from within-capacity to over-capacity is known as the El Farol Problem (see Arthur, 1994).
} 
Nash equilibria are also the socially optimal outcomes. For the battle-of-the-sexes game the Nash equilibria also correspond to the social optima, although they are the diagonal outcomes. Finally, our game requires $b>a=0$, and $2 b<1$ to ensure that the off-diagonal outcomes are efficient. Our game is the only one for which coordination is needed to reach a social optimum that is not an equilibrium. Private information further complicates this task by making it difficult to coordinate on any social optimum at all.

\section{Experimental Design and Procedures}

All experiments were conducted in fixed pairs. Each player in the pair received an independently and randomly drawn integer between 1 and 5 in each round. Subsequently, each player decided independently whether to enter or exit. The decision to exit yields 0 , whereas entry yields the value of the number if the opponent exits and $1 / 3$ of the value of the number if the opponent also enters. All experiments were conducted for 80 rounds.

At the end of each round, a player observes his opponent's decision to enter or exit. We conducted two experimental treatments that differ by the point in time at which a player learns his opponent's value (after the round or never). In "After", at the end of each round, each player learns his opponent's decision and value. In "Never", a player does not observe his opponent's number at the end of the round, only his decision to enter or exit.

The After treatment provides relatively favorable conditions for cooperation since the pair may coordinate on and enforce both alternation and cutoff strategies. If a player enters when it is not his turn to enter or on a low number, say 1 , he recognizes that his opponent will observe this defection and can retaliate by entering out of turn or the next time he receives a 1 .

In Never, deviations from alternating strategies are easily detected and punishable; whereas, defection from cooperative cutoff strategies is more difficult to detect. A player does not know if his opponent entered because he is playing uncooperatively or because he drew a high number. Frequent entry may just reflect lucky draws of high numbers. A rule could be adopted whereby 
more than 7 entries in the past 10 rounds constitutes a deviation; yet, efficiency would be lost if more than 7 of the last 10 draws exceeded the cutoff of 2.5. Furthermore, how does the pair coordinate upon the rule of 7 out of 10, or any other? This lack of information in Never renders cooperative cutoff strategies unlikely.

Upon arrival, each subject was seated in front of a computer terminal and handed the sheet of instructions (see Appendix B). After all subjects in the session had read the instructions, the experimenter read them aloud. To ensure full comprehension of the game, subjects were given a series of knowledge-testing questions about the game (Appendix B contains the questions). Participation in the experiment was contingent upon answering correctly all of the questions. ${ }^{5}$ Five practice rounds were then conducted with identical rules to the actual experiment. To minimize the influence of the practice rounds, subjects were rematched with a different opponent for the 80-round experiment.

An important feature of our experimental design that allows us to compare subjects' behavior across pairs and across treatments is our use of one pair of randomly drawn sequences of 80 numbers (85 numbers including the five practice rounds) from 1 to 5 . Before beginning the experiments, we drew two 80-round sequences, one for each pair member. We applied these sequences to all subject pairs in all sessions and treatments. ${ }^{6}$

We recruited subjects from a broad range of faculties and departments at Ben-Gurion University. Sixty-two subjects participated in one of the three After sessions and a different 62 subjects participated in one of the three Never sessions. In both treatments, one unit of experimental currency was exchanged for 0.6 shekels at the end of the session. A session lasted about 100 minutes on average, including the instructions phase and post-experiment questionnaire. Including a 10-shekel showup fee, the average subject profit was 76 shekels. $^{7}$

\footnotetext{
${ }^{5}$ No one was excluded from participating. All subjects who showed up answered correctly all of the questions in the allotted time.

${ }^{6}$ Thus, for instance, in round 56 regardless of pair, session or treatment, one pair member received a value of 2 , while the other received a value of 4 .

${ }^{7}$ One U.S. dollar equals about four shekels.
} 


\section{Cooperation in the Repeated Game}

Our theoretical results thus far all refer to the one-shot game where entry is the dominant strategy. However, the repetition of our experiments for 80 rounds with the same partner raises the possibility of some measure of cooperation. Indeed, in finitely repeated prisoners' dilemma and public goods games, cooperation is frequently observed before breaking down in the terminal rounds of play (see, e.g., Davis and Holt 1993). ${ }^{8}$

We can show that if subjects play the repeated game as if it is infinitely repeated, for a sufficiently high discount factor they will not deviate from the alternating strategy in After or Never nor from symmetric cooperative cutoff strategies in After.

Proposition 5: In an infinitely repeated game, for sufficiently patient players (high $\delta)$ :

(i) Alternation is a sequential-equilibrium (SE) strategy for the paired players in both the After and Never treatments;

(ii) The symmetric cooperative cutoffs $c=1.5, c^{*}=2.5$ and $c=3.5$ are additional SE strategies in the infinitely repeated game in the After treatment only;

(iii) It is possible to support cutoff cooperation as a SE in Never, but only when strategies consist of history-dependent actions.

From the proof of Proposition 5(iii), we observe that if cutoff cooperation is played, then the cutoff value responds dynamically to the player's history of values and entry decisions. Thus, to the extent that subjects in our experiments adopt these dynamic strategies, any attempt to categorize their play in Never as pure-strategy cutoffs will lead to higher error rates compared to After in which pure-strategy cutoffs may be supported in equilibrium.

\footnotetext{
8 Normann and Wallace (2004) compare cooperation in a finitely repeated prisoners' dilemma known to terminate after 22 rounds with two simulated infinitely repeated games with continuation probabilities of $1 / 6$ and $5 / 6$ implemented after round 22. Except for a significant endgame effect in the known finite-horizon game, the termination rule does not significantly affect cooperation rates. Dal Bo (2005) carefully calibrates the lengths of the finitely repeated games $(\mathrm{T}=2$ and 4$)$ to coincide with the expected lengths of the infinitely repeated ones $(\delta=1 / 2$ and $3 / 4)$ and finds higher cooperation rates in the comparable infinitely repeated games. Perhaps the finite horizons of two and four rounds are too short to encourage cooperation.
} 
The next proposition compares cooperation in After and Never.

Proposition 6: For any SE in Never, there is a SE in After with the same value-contingent payoffs; the reverse, however, is not true. ${ }^{9}$

The implication of Proposition 6 for our experiments is that, compared to Never, the subjects in After have a strictly larger set of SE upon which to coordinate. As a result, we anticipate higher cooperation in After than in Never.

Unconditional cooperation in humans is rare, in large part because it is easily exploited. Rather, individuals typically condition their cooperative behavior on their opponents' cooperating to a similar degree. Both the reward of cooperative behavior (positive reciprocity) and the punishment of uncooperative behavior (negative reciprocity) have been widely documented in the experimental literature (see, e.g., Andreoni et al. 2003, Fehr and Gachter 2000). In After, if a player's opponent is visibly playing, say, a higher cutoff than him, he may switch to the same cutoff and exit more frequently (positive reciprocity). Alternatively, he may respond to his opponent's deviations from a cutoff strategy by entering more frequently (negative reciprocity). The absence of information in Never inhibits subjects from directly rewarding or punishing numbercontingent actions. Instead, subjects may set a threshold of entry frequency for their opponent (e.g., 7 of the past 10 rounds, as mentioned in section 4); if the opponent exceeds this threshold, they respond by entering in one or more subsequent rounds regardless of their number.

With the promise of positive reciprocity or threat of negative reciprocity, a subject may willingly adopt this sort of entry budgeting. Jackson and Sonnenschein (2007) demonstrate that individuals may achieve higher social efficiency by budgeting (i.e., rationing) their representations in a voting game in accordance with their empirical distributions. However, Engelmann

\footnotetext{
${ }^{9}$ Proposition 6 resembles Kandori (1992) who shows that when players' actions are imperfectly observed by opponents, as the precision of the signal about an opponent's action increases, the set of sequential-equilibrium payoffs expands. In our framework, players' actions are perfectly observable; it is their payoffs from these actions that is private information. We have considered the two extremes of private information (Never and After). Instead, if players received a noisy signal about their opponents' payoffs after each round, then Proposition 6 would generalize to having the set of SE increase with the precision of the signal, parallel to Kandori.
} 
and Grimm (2006) show experimentally that only an exogenously imposed budget significantly improves the truthful representation of preferences and overall efficiency in this game.

Budgeting is not needed in After because play according to cutoffs is observable. Once equilibrium is reached, we would not anticipate negative reciprocity in After since it is off the equilibrium path. In Never, by contrast, optimal punishment may be set to deter deviation from a subject's budget when he receives low values. Notwithstanding, punishment may be insufficient to deter exceeding budget when the subject faces a $5 .^{10}$

\section{Results}

We begin this section by comparing cooperation across treatments. In 6.2 , we estimate whether a cutoff or the alternating strategy most closely characterizes each individual subject's observed decisions. Section 6.3 explores the dynamics in arriving at these strategies. We attempt to understand our results in 6.4 .

\subsection{Cooperation across Treatments}

Table 3 is a simple presentation of aggregate decisions: the entry percentage for a given number and treatment. For instance, in the After treatment when subjects drew the number 1, they entered only $16.3 \%$ of the time. These summary statistics reveal a number of findings. First, not all subjects are playing the static Nash equilibrium: exit is the modal decision for the number 1 in both treatments and also for the number 2 in After. Second, the sharp spike in entry percentages in going from the number 2 to 3 in both treatments suggests that many subjects may be employing the socially optimal cutoff of 2.5. Third, that not all subjects are entering all of the time on numbers 4 and 5, particularly in Never, suggests the use of alternating strategies for which entry and exit decisions are independent of the numbers received. Finally, as anticipated

\footnotetext{
10 The proof of Proposition 5(iii) provides an example.
} 
by Proposition 6, cooperation increases with increasing information, as seen by the overall lower entry frequency in After.

\section{[insert Table 3 here]}

To determine whether the difference in cooperation across treatments is significant we estimate a random effects Probit model on the pooled data from both treatments to explain the variation in subject $i$ 's decision to enter in period $t$. The specification for our random effects Probit model is as follows,

$$
\begin{aligned}
& \widetilde{\text { Enter }}_{i t}=\quad \begin{array}{l}
\text { constant }+\beta_{1} * \text { Never }+\beta_{2} * C_{1.5}+\beta_{3} * C_{2.5}+\beta_{4} * C_{3.5}+ \\
\\
\beta_{5} * C_{4.5}+\beta_{6} * \text { first } 10+\beta_{7} * \text { last } 10+\epsilon_{i t},
\end{array} \\
& \text { where } \quad \epsilon_{i t}=\alpha_{i}+u_{i t} \\
& \text { and } \text { Enter }_{i t}= \begin{cases}1 & \text { if } \widetilde{\text { Enter }_{i t}} \geq 0 \\
0 & \text { otherwise. }\end{cases}
\end{aligned}
$$

The treatment dummy Never tests for differences in the level of cooperation across treatments. The dummy variable $C_{1.5}$ equals one if player $i$ 's period $t$ number is $2,3,4$ or 5 and equals zero if it is 1 ; similarly, $C_{2.5}$ equals one for numbers 3,4 and 5 , and zero otherwise, and so forth for $C_{3.5}$ and $C_{4.5}$. The marginal effects of the estimated coefficients on these variables are interpreted as the marginal propensity to enter for numbers 2, 3, 4 and 5, respectively. Also in the regression equation are dummy variables for the first 10 and last 10 periods, which control for initial learning and end-game effects, respectively. The error term, $\epsilon_{i t}$, is composed of a random error, $u_{i t}$, and a subject-specific random effect, $\alpha_{i}$.

Table 4 displays the regression coefficients and marginal effects. The highly significant and positive coefficient on Never and the marginal estimate in regressions (1) and (2) indicate that a subject is about 5\% more likely to enter in Never than in After, controlling for the subject's number and for initial learning and end-game effects in (2). The significance of "first10" and 
"last10" supports these latter effects in the anticipated directions: subjects are less likely to enter early on and more likely to enter toward the end of the game.

$$
\text { [insert Table } 4 \text { here] }
$$

The estimates of $\rho$ in Table 4 measure the fraction of the error term's variance accounted for by subject-specific variance. The highly significant estimates of 0.192 and 0.411 indicate that about $19 \%$ and $40 \%$ of the variance in the error term is explained by subject heterogeneity in the two specifications, respectively.

\subsection{Individual Strategies}

To understand better the heterogeneity in subject behavior, we infer the strategy that best fits each subject's observed decisions, that is, the strategy that minimizes the number of errors in classifying the subject's decisions. We search over each of the possible pure-strategy cutoffs, $c \in\{0.5,1.5,2.5,3.5,4.5,5.5\}$, and the alternating strategy, generously modeled as the choice of an action opposite to the one made in the previous round. ${ }^{11}$

\section{[insert Table 5 here]}

Despite the slight payoff advantage and lower payoff variance of the alternating strategy, our main finding is the overwhelming adoption of cooperative cutoff strategies and the paucity of alternators. Table 5 reports the distribution of individuals' best-fit strategies by treatment for rounds $11-70 .{ }^{12} \quad$ The optimal symmetric cutoff strategy of $c^{*}=2.5$ best characterizes the decisions of $39 / 62$ subjects in the After treatment. ${ }^{13} \quad$ Nine subjects in After appear to be

\footnotetext{
11 Although there are other ways to model the alternating strategy, such as enter in odd or even rounds only, our chosen specification based on comparing decisions in rounds $t$ and $t-1$ is robust to mistakes: for instance, it detects subjects who began alternating, stopped for one or more rounds and resumed alternating by coordinating differently on who enters on the odd and even rounds.

12 Excluding the first 10 and last 10 rounds reduces the error rates by minimizing the initial learning and end-game effects documented in the regression analysis. The inferred best-fit strategies are highly robust to the different time horizons tested, like all 80 rounds, the last 60 rounds, the last 40 rounds and rounds 16-65.

${ }^{13}$ In the case where two strategies explain a subject's decisions equally well, each of the tied strategies receives half a point.
} 
employing the cutoff of 1.5; for one of these subjects, the static Nash strategy of always enter fits his decisions equally well. Eight additional subjects also play according to the Nash strategy of $c=0.5$, while four other subjects (two of whom form a pair) use the hyper-cooperative cutoff of 3.5. Only one pair of subjects alternates, beginning in period 33 and continuing without deviation through period 80 .

The Never treatment is a more likely candidate for alternating because the play of cutoff strategies cannot be observed or enforced. Still, a meager two out of 31 pairs alternate. ${ }^{14}$ An additional subject whose best-fit strategy is alternating eventually abandons it after his opponent fails to reciprocate.

Table 5 also reveals a marked shift from higher to lower entry cutoff values in going from After to Never. For example, the percentage of subjects playing the optimal symmetric pure-strategy cutoff declines from $62.1 \%$ in After to $38.7 \%$ in Never, while those who always enter increases from $13.7 \%$ to $20.2 \%$. Like the overall entry percentages in Table 3 and the regression results in Table 4, these individual inferred strategies also point to a decline in cooperation when less information is provided.

Overall, this inference technique fits the data well as seen in the error rates of $6 \%$ and $8 \%$ for the two treatments, respectively. ${ }^{15}$ Thus, of the 3720 decisions made by the 62 subjects in After between rounds 11 and 70, 3479 of them correspond to the best-fit strategy inferred for each subject. By comparison, if we assume that all subjects are playing the static Nash strategy, then the third-to-last row of data in Table 5 indicates that the error rates jump to $15 \%$ and $32 \%$ depending on the treatment. In addition, we generated random decisions for subjects calibrating the probability of entry to match the observed overall rate of entry in each treatment (.677 and .744 respectively for the treatments). We then calculated the error rate from these random decisions for each subject's best-fitting strategy and for each subject assuming static Nash play. The results in the bottom two rows of Table 5 again demonstrate that our inferred

\footnotetext{
${ }^{14}$ For these pairs, alternation begins in rounds 2 and 8, respectively, and continues flawlessly for the duration.

15 The higher error rate for Never agrees with the implication of Proposition 5.
} 
strategies on the actual data fit the data much better than the best-fitting and Nash strategies on the randomly generated data. In sum, subjects are indeed playing in a non-random, methodic fashion that can be captured mostly by cutoff strategies. ${ }^{16}$

Our strategy analysis also reveals that pair members typically coordinate on the same cooperative strategy. In After, of the 30 pairs that employ cutoff strategies, members in 22 pairs coordinate on the same cutoff values. If subjects independently chose their strategies according to the observed distribution of best-fit strategies, the probability of at least 22 pairs coordinating on the same cutoff is $1 / 60$. Sixteen out of 28 pairs do so in Never. In addition, for all 12 pairs in which partners do not coordinate on the same cutoffs, their inferred cutoffs differ by only one integer value. Again, if subjects drew their strategies independently from the observed distribution, the likelihood of obtaining this degree of coordination or better is $1 / 600$. The implication is that it is extremely unlikely in both After and Never that paired subjects achieved the observed degree of coordination on the same or similar cutoff values by mere chance. Paired subjects' coordination is particularly surprising in Never, since opponents' values are unobservable.

Successful coordination on the same strategy shows up in the form of higher profits for paired subjects playing the optimal cooperative cutoff strategy than those who always enter. The righthand columns of each treatment in Table 5 reveal that the average subject profits for $c^{*}=2.5$ are 111.6 and 106.8 in After and Never, respectively, compared to 109.5 and 93.2 for always enter. These profits reveal that non-cooperators earned substantially more in After than in Never. The explanation is that 5/9 Nash players in After were paired against more cooperative subjects compared to only $3 / 13$ in Never. How did such a relatively high fraction of subjects in After allow their partners to always enter, when entry on values of 1 and 2 is perfectly observable in this treatment? A likely explanation is that these unconditional cooperators encourage defection. A subject who enters on a 1 and observes that his opponent does not retaliate is emboldened to

\footnotetext{
16 A complementary method to determine subjects' strategies is to ask them. We did this in a post-experiment questionnaire. Many subjects claim to decide randomly when in fact their decisions display a clear tendency to enter on higher numbers and exit on lower ones. For the minority of subjects whose responses are interpretable as either alternating or cutoffs, they match our inferred strategies well.
} 
do so again, while in Never the defector as such is not observable.

\subsection{Dynamics}

The strategy analysis in the preceding section does not help us understand how pair members succeed in coordinating on the same cutoff values. In this section, we explore two forms of dynamic behavior that may have aided coordination, budgeting and reciprocity (both discussed in section 5).

The idea underlying budgeting is that a player bases his current-period entry decision on his entry frequency in previous periods. The player has a target frequency of entry from which he attempts not to deviate. Having entered frequently in recent periods, he may choose to exit in the current period (regardless of his value) to avoid exceeding this target. We refer to this as "gentlemanly budgeting". The maintenance of goodwill with one's partner may motivate gentlemanly budgeting. On the other hand, if the player has exited frequently in recent periods, he may choose to enter in the current period (regardless of his value) to maintain his target entry frequency. We refer to this as "opportunistic budgeting".

Consider, for example, a subject with a target entry frequency of $60 \%$. In an effort not to deviate excessively from this target, the subject may decide to enter if he has exited in six or more of the past 10 periods and to exit if he has entered in eight or more of the last 10 periods. Alternatively, the subject may base his current-period entry decision on his entry frequency from the past five or past three periods only. These examples begin to illustrate the large number of possible time horizons and rules for entry and exit on which subjects may condition their entry decisions.

We do not aspire to understand individual subjects' time horizons or target entry frequencies. Instead, to determine empirically whether the gentlemanly or opportunistic budgeting motives may guide subjects' entry decisions, we include two dummy variables in estimating subject $i$ 's entry decision in period $t$ : "iprevious2enter" assumes a value of 1 if the subject entered in the 
previous two rounds and zero otherwise; similarly, "iprevious2exit" takes on a value of 1 if the subject exited in the previous two rounds and zero otherwise. Looking at occurrences where the subject makes the same decision in the previous two rounds both eliminates alternators and captures budgeting over two or more rounds. ${ }^{17}$

We also explore empirically the reciprocity motive. A subject may reward his opponent's recent under-entry by exiting in the current period (positive reciprocity). Similarly, he may punish his opponent's recent over-entry by entering (negative reciprocity). Again to avoid confounding alternating with reciprocity, we investigate whether the subject is responsive to his opponent's decisions made in the past two periods. The variable "uprevious2enter" takes on a value of 1 if the opponent entered in periods $t-1$ and $t-2$, and zero otherwise. The variable "uprevious2exit' is analogously defined. As with budgeting, these variables capture reciprocity based on the opponent's decisions from the past two or more rounds.

Table 6 reports the results of these random-effects regressions designed to test for the budgeting and reciprocity motives in the After and Never treatments. From regression (1), two significant tendencies can be seen in After: subjects are less likely to enter in the current round if either in the previous two rounds they entered or their opponent exited (iprevious2enter and uprevious2exit are both negative and significant). The first of these trends points to gentlemanly budgeting, while the latter suggests positive reciprocity. Interestingly, from regression (3) we see that neither of these two variables is significant in Never, whereas precisely the other two variables (iprevious2exit and uprevious2enter) are both highly significant and positive. That is, subjects are more likely to enter in the current round if either in the previous two rounds they exited or their opponent entered. The first of these trends is opportunistic budgeting, while the latter reflects negative reciprocity.

\section{[insert Table 6 here]}

\footnotetext{
17 For example, if for rounds one to seven the subject has a budget of entering up to four times, then entry in, say, rounds 1 and 2 increases the threshold value (and therefore decreases the likelihood) of entry in round 3, whereas consecutive exiting in rounds 1 and 2 decreases the threshold entry value in round 3.
} 
Both of these budgeting and reciprocity trends in Never are less cooperative and imply more entry than the corresponding findings in After. ${ }^{18}$ By taking advantage of the unobservability of values in Never to enter when they have exited "too often", subjects exhibit limited trustworthiness. By entering when their opponent has entered "too often", subjects exhibit limited trust that their opponent is abiding by their implicitly agreed upon cutoff strategies.

To determine whether these budgeting and reciprocity tendencies take place simultaneously or at different stages during the course of play, in regressions (2) and (4) we interact each of the four variables from (1) and (3), respectively, with a dummy for the first 40 rounds ("half1") and the last 40 rounds ("half2"). In After, gentlemanly budgeting plays a significant role only in the first half as subjects push for cooperation while they learn to play a cutoff strategy. The weakly significant negative coefficient on "uprevious2exit*half2" is evidence of positive reciprocity in the latter half of the game and reflects the subject's willingness to be pulled to more cooperative play. ${ }^{19}$ In Never, opportunistic budgeting is a significant motive throughout, whereas negative reciprocity is significant in the latter half only as subjects attempt to maintain cooperation.

\subsection{Why so few alternators?}

Although the pair's expected profit from alternating is slightly higher (by 0.12 units per period) and the variance lower than those from the optimal cutoff strategy of $c^{*}=2.5$, the overwhelming majority of subjects employ cutoffs. There are two possible explanations.

First, successful alternation requires coordination on the part of both pair members, as witnessed by several subjects who began the game alternating, but eventually abandoned it after their partner failed to reciprocate. By contrast, cutoff cooperation can be implemented unilaterally and its usefulness does not require coordination on the same cutoff.

\footnotetext{
18 If we replace these four variables based on the same previous two-round decisions with analogous variables from the previous three rounds, all of our findings above as well as those reported below remain qualitatively similar.

19 Once in equilibrium, neither reciprocity nor budgeting is necessary in After, according to our theory in section 5. The significance of these coefficients indicates a transition.
} 
Second, cutoff cooperation is cheap, since it involves exiting on the lowest values, when it is least costly to do so; whereas, alternation ignores the value to entry. Thus, for a player who decides to cooperate regardless of whether his opponent reciprocates, his foregone profit from exiting only on low values is less than if he exits just as often without regard for values (as with alternating).

To determine which of these reasons accounts for subjects' unwillingness to alternate, we designed an additional pair of treatments that maintains the difficulty of coordinating jointly on the alternating strategy, but makes cutoff cooperation less cheap.

\section{$7 \quad$ In Search of Alternating}

\subsection{Experimental Game and Procedures}

By shrinking the percentage difference between $\underline{v}$ and $\bar{v}$, the values to entering become more alike making cutoff cooperation less cheap. In the extreme case where $\underline{v}=\bar{v}$, one would expect all players to alternate. If siblings derive identical utility from riding in the front seat, they will take turns enjoying this privilege.

To determine if a game parameterization with more similar values can increase alternation, we conducted two additional treatments. We added a constant of 100 to the randomly drawn numbers 1-5. Thus, the After100 and Never100 treatments are identical to the similarly named original treatments (i.e., five integers in the range, $\mathrm{k}=3$ ), except that players' iid integers come from the uniform distribution 101 to 105 . The dominant stage-game strategy remains entry. ${ }^{20}$ By corollary 3, we know that the socially optimal cutoff involves exiting on at least the lowest integer in the range. Indeed, $c^{*}=101.5$ is the socially optimal pure-strategy cutoff and yields the pair an expected payoff of 77.28 units per round. By comparison, alternating earns 103 in expectation, a $33 \%$ premium over $c^{*}=101.5$. The research question can then be phrased as,

\footnotetext{
${ }^{20}$ Note well that the payoff to exit remains zero. If we had also added 100 to the exit payoff, entry would no longer be the dominant strategy.
} 
is the joint payoff premium to alternation sufficient to overcome its inherent dual coordination problem?

Sixty and 70 subjects participated in After100 and Never100, respectively. Participation was again restricted to one experiment per subject and no subject had participated in either After or Never. In selecting the experimental-currency-to-shekel ratio, we held constant across both sets of treatments the joint monetary payoff from the optimal cooperative strategy of alternating. Also, keeping fixed his opponent's decisions, a player always entering earns the same expected monetary payoff in all treatments. This implies a new exchange rate of 1:0.0175. Table 7 compares the nominal and real payoffs in the original and follow-up treatments.

[insert Table 7 here]

\subsection{Results from Follow-Up Treatments}

The last two columns of Table 8 reveal little variation in the entry decisions as a function of the number received in both After100 and Never100. Overall, about $61 \%$ of the decisions are enter on 101, increasing to $67 \%$ on the number 105 . This stability of entry percentages attests to alternation. The strategy inference analysis in Table 9 confirms the preponderance of alternators: alternating is the best-fit strategy for $64 \%$ of subjects in Never 100 and a still higher $73 \%$ of subjects in After100, even though After100 affords the opportunity to observe and thus coordinate on cooperative cutoff strategies. Alternating and Nash play account for about 95\% of subjects in both treatments. No subject plays according to the optimal cutoff $c^{*}=101.5$ in After100 and only 1.5/70 adopt this cutoff in Never100. Put starkly, those who cooperate in these experiments alternate; the remaining quarter of the subjects are best described by entering in every round.

$$
\text { [insert Tables } 8 \text { and } 9 \text { here] }
$$

How can we explain the shift from almost all cutoff cooperators in the original treatments to almost all alternators in these follow-up treatments? By adding a constant of 100, both the 
nominal and real payoffs to entry are made very similar for all values 101-105, as shown in Table 7. It no longer matters substantively whether a player enters alone on the highest or lowest integer in the range: the difference in both monetary and nominal terms between the two outcomes is a paltry $4 \%$ compared to $400 \%$ in the original treatments. In addition, Table 7 shows that exiting on the lowest integer is about three times costlier in these treatments than the original ones, no matter if only one player exits (foregone profit of 0.588 vs. 0.2 ) or both do (foregone profit of 1.765 vs. 0.6). Alternation ensures that exactly one player enters each round, thereby at once avoiding congestion and the now costlier outcome whereby both players receive low draws and exit.

The prevalence of alternators in these treatments implies that the two-person coordination problem inherent in alternating is not insurmountable. By increasing the joint-expected-payoff advantage to alternating (i.e., by making cutoff cooperation less cheap), all cooperators switch from cutoffs to taking turns.

Figures $2 \mathrm{a}$ and $2 \mathrm{~b}$ also attest to the pervasiveness and stability over time of the alternating strategy in both After100 and Never100. If all subjects employed the alternating strategy throughout the game, $50 \%$ of the decisions would be exit in every round; whereas exit percentages varying from 0 to 100 depending on the numbers drawn would reflect cooperative cutoff strategies. In round 3, for example, paired subjects drew numbers 101 and 102 followed by 104 and 105 in round 4. Accordingly, the exit percentage swung from 57\% to $17 \%$ in Never 100 and from $48 \%$ to $23 \%$ in After100, suggesting that some subjects are using cooperative cutoffs in these early rounds. In After100, from round 17 through round 78, the percentage of exit decisions stabilizes at about 40\%, despite the randomly drawn numbers each round. In Never100, the percentage of exit decisions starts below $30 \%$ and it is not until round 40 that it reaches $40 \%$ where it stabilizes, again until the second-to-last round. ${ }^{21}$

[insert Figures 2a and 2b here]

\footnotetext{
21 The relatively high error rate of the best-fit strategies of 0.13 in Never100 compared to only 0.06 in After 100 also reflects the extra time required to converge on alternating in Never100.
} 
It is surprising that After100 reveals a higher percentage of alternating pairs and their faster formation than Never100 where alternating is the only verifiable cooperative strategy.

How can we understand this opposite finding? It turns out that learning the opponent's number at the end of the round facilitates the formation of alternating pairs even though alternating in no way depends on the subject's number. A subject in After100 who exits on a 104 or 105 while entering on a 101 or 102 in other rounds sends a strong signal that he is not playing a cutoff strategy. Never100 offers no such conspicuous opportunity to communicate one's intentions due to the unobservability of the opponent's number.

The other striking observation from these time series of exit decisions is the sudden drop off in exiting in the final two rounds. In After100, from around $40 \%$ of all decisions in rounds $17-78$, exit decisions plummet to $23 \%$ and $5 \%$ in rounds 79 and 80 , respectively. Similarly, in Never 100 , exit decisions fall to $26 \%$ and $7 \%$ in the last two rounds after having stabilized at around $40 \%$ during the last half of the game. Like the endgame effects observed in the original treatments, these sharp declines in cooperative behavior in the final two rounds suggest that cooperation throughout the game is at least in some measure strategic.

\section{Lessons for Cooperation in the Real World}

Cooperation assumes many forms in the real world. We design a class of games that permits two distinct forms of cooperation in the repeated game. These games are characterized by a tension between the unique static Nash equilibrium in which both players enter and the socially optimal outcome in which one player enters and the other exits. We derive the theoretical properties of these games and optimally select a parameterization to study experimentally. The asymmetric social optimum permits alternating as one cooperative repeated-game strategy. Privately known, randomly drawn values for entering admit cutoff strategies as another form of cooperation.

We find that when the range of values to cooperation is similar, players alternate; whereas, diffuse payoff distributions enhance the value to private payoff information and lead to the 
adoption of cutoff strategies. In real-world cooperation dilemmas, participants' values tend to be alike and thus, according to our results, alternation adopted, when the cooperative task is mundane or requires little skill. By contrast, individuals' values are varied for tasks that require a particular, unequally distributed skill or tasks that elicit a strong heterogeneity in preferences. In these situations, our results reveal that cooperative participants will adopt cutoff strategies, even if alternation yields similar expected payoffs. For participants to overcome the costly twoperson coordination required of alternation, it must provide a substantially higher payoff than cutoff strategies.

Several examples illustrate these distinctions. Cabdrivers are assigned to passengers in two distinct ways. Similar to alternating, cabdrivers at a taxistand serve passengers in a predetermined order based on the observable time at which the drivers returned to the stand. This solution distributes passengers evenly and prevents conflict between cabbies, all of whom are available with identical locations. However, for passengers who call the cab company, such an assignment would be inefficient and likely to elicit complaints from passengers and from the cabbies dispersed throughout the city. Instead, the dispatcher broadcasts the location of the passenger and allows cabdrivers to take advantage of their private information (e.g., availability, distance to the customer) to divide up customers according to their values, similar to cutoff strategies.

Likewise, an army sergeant, shift manager, head of a team of programmers or committee of commune members may assign mundane tasks (e.g., cleaning the latrine, unpopular shifts, routine programming) using a duty roster or other system that disregards input from group members (alternating); or, in the case of diverse values (e.g., combat duty, revenue-generating activities for the commune, choice shifts, challenging programming), the members may self-select their tasks based on privately known ability and preferences (cutoffs).

Allowing group members to choose their tasks exploits their private information. By the same token, conflict may ensue if more than one member opts for the same task, while other unwanted tasks may remain unfilled. Alternation assigns exactly one member to each task, thus at once 
avoiding conflict and ensuring that no opportunity is missed, while at the same time conveying an impression of an equitable distribution of assignments.

\section{References}

Andreoni, James, William Harbaugh and Lise Vesterlund (2003) "The Carrot or the Stick: Rewards, Punishments, and Cooperation," American Economic Review, 93:3, 893-902.

Arthur, W. Brian (1994) "Inductive Reasoning and Bounded Rationality," American Economic Review Papers and Proceedings, 84:2, 406-411.

Dal Bo, Pedro (2005) "Cooperation under the Shadow of the Future: Experimental Evidence from infinitely Repeated Games," American Economic Review, 95:5, 1591-1604.

Davis, Douglas and Charles Holt (1993) Experimental Economics, Princeton University Press, Princeton, NJ.

Engelmann, Dirk and Veronika Grimm (2006) "Overcoming Incentive Constraints? The (In-) Effectiveness of Social Interaction," unpublished manuscript.

Fehr, Ernst and Simon Gachter (2000) "Cooperation and Punishment in Public Goods Experiments," American Economic Review, 90:4, 980-994.

Jackson, Matthew and Hugo Sonnenschein (2007) "Overcoming Incentive Constraints," Econometrica, 75:1, 241-57.

Kahneman, Daniel (1988) "Experimental Economics: A Psychological Perspective," R. Tietz, W. Albers and R. Selten (eds.), Bounded Rational Behavior in Experimental Games and Markets, Berlin: Springer-Verlag, 11-18.

Kandori, Michihiro (1992) "The Use of Information in Repeated Games with Imperfect Monitoring," Review of Economic Studies, 59:3, 581-593.

Normann, Hans-Theo and Brian Wallace (2004) "The Impact of the Termination Rule in Cooperation Experiments," unpublished manuscript.

Rapoport, Amnon (1995) "Individual strategies in a market entry game," Group Decision and Negotiation, 4, 117-133.

Rapoport, Amnon, Darryl A. Seale and Eyal Winter (2002) "Coordination and Learning Behavior in Large Groups with Asymmetric Players," Games and Economic Behavior, 39, 111-136. 
Rapoport, Amnon, Darryl A. Seale and Eyal Winter (2000) "An Experimental Study of Coordination and Learning in Iterated Two-Market Entry Games," Economic Theory, 16, 661-687.

Van de Kragt, A., R. Dawes and J. Orbell (1983) "The Minimal Contributing Set as a Solution to Public Goods Problems," American Political Science Review, 77, 112-122.

Zillante, Artie (2005) "Spaced-Out Monopolies: Theory and Empirics of Alternating Product Release," unpublished manuscript.

\section{Appendix A - Proofs of Propositions}

Proof of Proposition 2: Let us examine the costs and benefits of extending the symmetric cutoff by one from $c-1 / 2$ to $c+1 / 2$. We can represent the problem on a grid that is $\bar{v}-\underline{v}+1$ units by $\bar{v}-\underline{v}+1$ units. Each point on the grid refers to the net gains if the numbers drawn are from that point. The uniform independent distribution implies that each grid point has equal weight. Let us refer to each point as $(x, y)$. The points affected are $(\cdot, c)$ and $(c, \cdot)$. Divide this set of points into three groups. Group one is $(c, z)$ and $(z, c)$ where $z>c$. Group two is $(c, z)$ and $(z, c)$ where $z<c$. Group three is $(c, c)$.

For each grid point in group one, there is a net gain of $z-(z+c) / k$. For group two, there is a net loss of $c$ for each grid point. For group three, there is a net loss of $2 c / k$. For all of the points together, there is a net gain of,

$\underbrace{2 \sum_{z=c+1}^{\bar{v}}\left(z-\frac{z+c}{k}\right)}_{\text {Group } 1}-\underbrace{c \cdot 2(c-\underline{v})}_{\text {Group } 2}-\underbrace{2 \frac{c}{k}}_{\text {Group } 3}=\frac{\bar{v}(1+\bar{v})(k-1)-(1+2 \bar{v}+k-2 \underline{v} k) c-3(k-1) c^{2}}{k}$.

This is simply a quadratic with both a positive and a negative root. The expression represents the net benefit of increasing the pure-strategy cutoff $c$ starting at $\underline{v}$. It will eventually become negative as $c$ surpasses the positive root $c^{*}$. Thus, for pure strategies, the positive root provides the optimal cutoff where exiting occurs if and only if one's number is below $c^{*}$. From Lemma 1 , the optimal cutoff strategy may entail the use of mixed strategies. While we don't do so here, the optimal mixed strategy can be derived in the same manner.

$Q E D$ 
Proof of Corollary 3: The two comparative-statics results preceding the corollary indicate that the most difficult test for the corollary is $k=3$ and the range of integers $[\underline{v}, \underline{v}+1]$. If we can show that the socially optimal strategy is exit on $\underline{v}$ for this case, then the corollary holds for all $\bar{v}>\underline{v}$ and $k \geq 3$. Each player earns in expectation $\left(\frac{v}{2}+\frac{\underline{v}+1}{2}\right) \cdot \frac{1}{3}=\frac{2 \underline{v}+1}{6}$ if he enters on both $\underline{v}$ and $\underline{v}+1$. But by staying out on $\underline{v}$, each player does better with expected earnings equal to $\frac{v+1}{2} \cdot\left(\frac{1}{2}+\frac{1}{2} \cdot \frac{1}{3}\right)=\frac{2 v+2}{6}$.

Proof of Proposition 4: Independent of $k$, alternating yields a joint expected payoff equal to the expected value of the range of numbers, while the joint expected payoff of any symmetric cutoff strategy (strictly less than $\bar{v}$ ) is strictly decreasing in $k$. Consider the case of $k=2$. The strategy of always enter (the lowest possible cutoff) yields half the expected value for each player. Thus, for $k=2$, the joint expected payoffs are the same for alternating and always entering. Hence, for $k<2$, the joint expected payoff from the not necessarily optimal cutoff strategy of always enter will be strictly higher than that of alternating, implying that the optimal cutoff will yield expected payoffs that are strictly higher as well.

For the uniform distribution and $k \rightarrow \infty$, using the grid method of the previous proof, alternating yields $(\bar{v}-\underline{v}+1) \sum_{z=\underline{v}}^{\bar{v}} z=(1+\bar{v}-\underline{v})^{2}(\underline{v}+\bar{v}) / 2$. As $k \rightarrow \infty$, both players entering yields a payoff of 0 (as does both exiting). This simplifies the joint-payoff calculation of the cutoff strategy $c^{*}$ to $2\left(c^{*}-\underline{v}\right) \sum_{z=c^{*}}^{\bar{v}} z=\left(1+\bar{v}-c^{*}\right)\left(c^{*}-\underline{v}\right)\left(\bar{v}+c^{*}\right)$. The expression $\left(1+\bar{v}-c^{*}\right)\left(c^{*}-\underline{v}\right)$ reaches its maximum at $c^{*}=(1+\underline{v}+\bar{v}) / 2$, yielding $(1+\bar{v}-\underline{v})^{2} / 4$. Since $\left(\bar{v}+c^{*}\right)$ is maximized for $c^{*}=\bar{v}$, we know the joint cutoff payoff must be strictly less than $(1+\bar{v}-\underline{v})^{2} \cdot \bar{v} / 2$. For $\underline{v}>0$, this is less than the joint alternating payoff.

Proof of Proposition 5: (i) Assume that a deviation from the intended strategy prompts the opponent to enter forever after (grim trigger strategy). Such a deviation from alternation is done by entering out of turn and always detected in either the After or Never treatments. The payoff to deviating from alternation is highest on a 5 and yields an expected payoff of, $\frac{5}{3}+1 \cdot \frac{\delta}{1-\delta}$, 
whereas continued alternation yields an expected payoff of $3 \delta+3 \delta^{3}+\ldots=\frac{3 \delta}{1-\delta^{2}}$. Thus, a player will never deviate from alternation if and only if $\frac{3 \delta}{1-\delta^{2}} \geq \frac{5}{3}+\frac{\delta}{1-\delta}$, or $\delta \geq 0.679$.

(ii) Again assuming a trigger-strategy punishment, we now verify that each of the cooperative cutoffs $c=1.5, c^{*}=2.5$ and $c=3.5$ constitutes a symmetric SE for a sufficiently high $\delta$. For each of these cutoff strategies, the most profitable deviation involves entering on the highest value below the cutoff, namely, entering on $c-0.5$. The expected present discounted deviation payoff is thus, $(c-0.5)(1-p)+\frac{c-0.5}{3} p+\frac{\delta}{1-\delta}$, where $p$ is the probability that the opponent also enters in the deviation round, equal to $0.8,0.6$ and 0.4 when $c=1.5, c^{*}=2.5$ and $c=3.5$, respectively. The expected present discounted payoff from $c=1.5, c^{*}=2.5$ and $c=3.5$ for the infinitely repeated game are $\frac{1.305 \cdot \delta}{1-\delta}, \frac{1.44 \cdot \delta}{1-\delta}$ and $\frac{1.32 \cdot \delta}{1-\delta}$, respectively. Comparing each of these expressions with the corresponding deviation payoff implies that a player never deviates as long as $\delta \geq 0.605$ for $c=1.5, \delta \geq 0.732$ for $c^{*}=2.5$, and $\delta \geq 0.873$ for $c=3.5$. In addition, the cooperative cutoffs 4.5 and 5.5 cannot be supported as symmetric SE since they yield lower stage-game joint expected payoffs than always enter (see Table 1).

In Never, these symmetric cooperative cutoffs cannot form an equilibrium strategy. Consider the following two sets of realizations of infinite sequences of random values, $\{x, y\}$ and $\left\{x^{\prime}, y^{\prime}\right\}$. For both sequences, player 1 has the exact same realizations, $x=x^{\prime}$. Player 2's realizations differ in round $t$ only: in one sequence he draws a $5, y_{t}=5$, while in the other he draws a $1, y_{t}^{\prime}=1$. In all other rounds $s \neq t$, we have $y_{s}=y_{s}^{\prime}$. When $y_{t}^{\prime}=1$, we have shown that the equilibrium strategy in After involves exiting, since entry triggers player 1's punishment strategy. In Never, the two sequences $\{x, y\}$ and $\left\{x^{\prime}, y^{\prime}\right\}$ are indistinguishable to player 1 so that play by a cutoff strategy dictates the same actions for player 1 . Hence, if player 2 plays as if he drew sequence $y$ when in fact he faces sequence $y^{\prime}$, player 2 profits from this deviation simply because player 1 behaves exactly as if player 1 drew sequence $y$.

(iii) In part (ii) we already established that non-history dependent cutoff cooperation is not sustainable. Here we establish that a form of cutoff cooperation is indeed possible. Consider 
the cooperative strategy of exiting one out of every two rounds. Let us assume that as opposed to alternating, this is done solely by cutoffs. For example, on a 1 or 2 in the first round, a player stays out in the first round and enters in the second. On a 3, 4 or 5, a player enters in the first round and stays out in the second. This gives a two-round expected payoff of $0.6 \cdot(4 \cdot 0.4+4 / 3 \cdot 0.6)+0.4 \delta \cdot(3 \cdot 0.6+1 \cdot 0.4)=1.44+0.88 \delta$. Let us again assume triggerstrategy punishment. The highest incentive to deviate occurs when one player draws a 5 in the second round, but knows that both players are supposed to stay out in that round. The payoff to deviating is $5+\frac{\delta}{1-\delta}$. The payoff for staying the course is $\frac{\delta(1.44+0.88 \delta)}{1-\delta^{2}}$. Cooperation can be established if $\delta>0.968$.

$Q E D$

Proof of Proposition 6: Any SE in Never for which the equilibrium strategies are contingent on observables can be exactly duplicated in After. What if the equilibrium strategies in Never condition on player $i$ 's own value, which is unobservable to the opponent? We will show that for these equilibria, there are comparable, isomorphic equilibria in After in which the player's value-contingent strategy can be replaced with a value-independent strategy that attains the same expected action profile and the other player chooses the same strategy as in Never; as a result, both players earn the same expected payoffs as in Never.

Consider player $i$ 's strategy in period $t$ denoted by $s_{i t}\left(v^{t-1}, v_{i t}, a^{t-1}\right)$, where $v^{t}$ and $a^{t}$ are the histories of all values and actions, respectively, up to period $t$. In Never, since the opponent's values are never revealed, a player's strategy can condition on his own values only, $s_{i t}\left(v_{i}^{t}, a^{t-1}\right)$. In Never, player $i$ 's present and future payoffs depend on his previous values only insofar as they affect his individual strategy. This is because his period payoff is $u_{i}\left(v_{i t}, a_{i t}, a_{j t}\right), j \neq i$, and only his actions can depend upon previous values. In After, the opponent's j's action can also depend on player $i$ 's values, $i \neq j$.

For any Never equilibrium in which player $i$ 's strategy depends upon his previous values, there exists another equilibrium that yields the same expected payoffs for both players where player $i$ 's strategy does not depend upon his previous values and the other player employs the same 
strategies as in the initial equilibrium. This holds because player $i$ must be indifferent between his value-dependent strategy and one that isn't dependent on previous values that mixes with the same action probabilities, that is, $P\left(a_{i t}=A \mid a^{t-1}, v_{i t}\right)$ is the same for both strategies. This value-independent strategy must be an equilibrium since it does not affect the other player's expected payoffs. These same value-independent strategies form an equilibrium in After. QED

We illustrate this equivalence with an example. Suppose the history $a^{t-1}$ in which $a_{i t-1}=$ enter forms part of an equilibrium in Never. Suppose also that player $i$ enters in round $t$ for $v_{i t}$ equal to 4 or 5 , and enters on value 3 if and only if $v_{i t-1}=4$. An equivalent equilibrium strategy for this period would be to enter on a 3 with probability $P\left(a_{i t}=\right.$ enter $\left.\mid a^{t-1}, v_{i t-1}=4\right) \equiv 1 / 3$. Applying this to both players in all periods generates an equilibrium based on observables in both Never and After.

The fact that the symmetric cutoff of $c^{*}=2.5$ can be a SE in After (Proposition 5ii) but not Never serves as a counterexample to establish the second part of the proposition, namely, that not all SE in After are also in Never.

\section{Appendix B - Participants' Instructions}

\section{Pre-Experiment Questions (not intended for publication)}

1. How many numbers are there in the range of 1 to 5 ?

2. What is the probability of obtaining the number "4" in any given round?

3 . What is the anticipated average of the numbers you will receive over the entire 80 rounds of play? 4. Suppose that you have received the number "1" during three consecutive rounds. What is the probability of receiving another" 1 " in the next round?

5. Suppose that you receive the number "1" and your opponent receives a "2" in a particular round. a. If you both enter, what will be your payoff from this round? What will be your opponent's payoff? b. If you enter and your opponent exits, what will be your payoff from this round? What will be your opponent's payoff?

c. If you both exit, what will be your payoff from this round? What will be your opponent's payoff?

d. If you exit and your opponent enters, what will be your payoff from this round? What will be your opponent's payoff? 


\begin{tabular}{cc|c|c|} 
& & \multicolumn{2}{|c|}{ Other Person } \\
& & Enter & Exit \\
\hline \multirow{2}{*}{ You } & Enter & $\mathrm{x} / 3, \mathrm{y} / 3$ & $\mathrm{x}, 0$ \\
& Exit & $0, \mathrm{y}$ & 0,0 \\
\hline
\end{tabular}

\section{Instructions to Participant (After treatment)}

The experiment in which you will participate involves the study of decision-making. The instructions are simple. If you follow them carefully and make wise decisions, you may earn a considerable amount of money. Your earnings depend on your decisions. All of your decisions will remain anonymous and will be collected through a computer network. Your choices are to be made at the computer at which you are seated. Your earnings will be revealed to you as they accumulate during the course of the experiment. Your total earnings will be paid to you, in cash, at the end of the experiment.

There are several experiments of the same type taking place at the same time in this room.

This experiment consists of 80 rounds. You will be paired with another person. This person will remain the same for all 80 rounds. Each round consists of the following sequence of events. At the beginning of the round, you and the person with whom you are paired each receives a randomly and independently drawn integer number from 1 to 5 inclusive. This number is your private information, that is, the other person will not see your number and you will not see the other person's number. After seeing your numbers, each of you must decide separately between one of two actions: enter or exit. At the end of each round, your number, your action, and the other person's action determine your round profit in the following way. If you both chose to exit, then you both receive zero points. If you chose to exit and the other person chose to enter, then you receive zero points and the other person receives points equal to his number. On the other hand, if you chose to enter and the other person chose to exit, you receive points equal to your number and the other person receives zero points. If you both chose to enter, then you receive points equal to one-third of your number and the other person receives points equal to one-third of his number. The table below summarizes the payoff structure.

Suppose you receive a number, $\mathrm{x}$, and the other person receives a number, $\mathrm{y}$. The round profits for each of the given pair of decisions are indicated in the table below. The number preceding the comma refers to your round profit; the number after the comma is the other person's round profit.

After you have both made your decisions for the round, you will see the amount of points you have earned for the round, the other person's decision and his number. When you are ready to begin the next round, press Next.

At the end of the experiment, you will be paid your accumulated earnings from the experiment in cash. While the earnings are being counted, you will be asked to complete a questionnaire. Prior to the beginning of the experiment, you will partake in a number of practice rounds. The rules of the practice rounds are identical to those of the experiment in which you will participate. Note well that for the purpose of the practice rounds, you will be paired with a different person from the actual experiment. The purpose of the practice rounds is to familiarize you with the rules of the experiment and the computer interface. The profits earned in these practice rounds will not be included in your payment. If you have any questions, raise your hand and a monitor will assist you. It is important that you understand the instructions. Misunderstandings can result in losses in profit. 
Figure 1

Plot of joint expected payoff as a function of symmetric cutoff and $k$

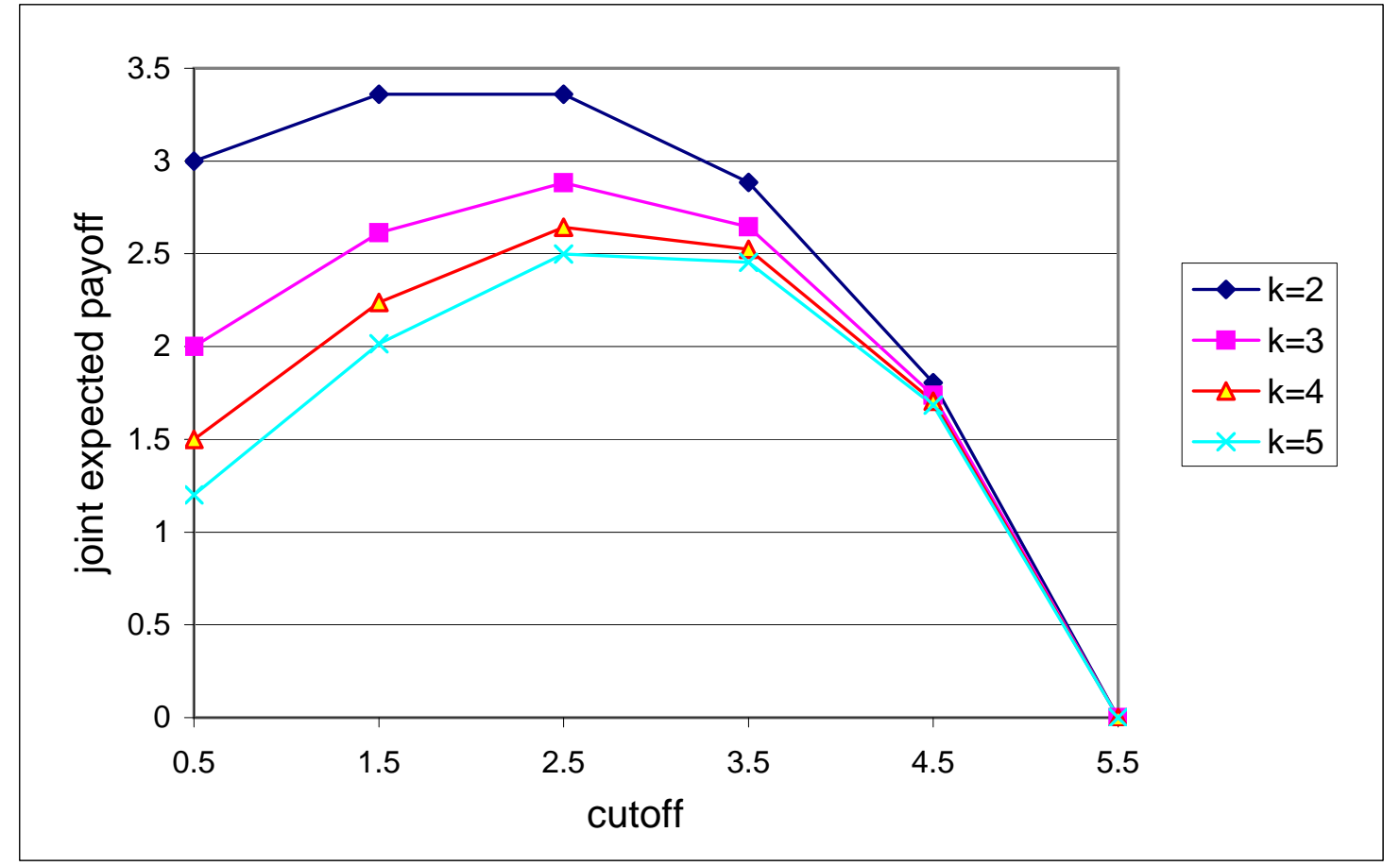

The pair's joint expected payoff as a function of symmetric pure-strategy cutoffs 0.5 to 5.5 for the range of numbers 1 to 5 and the indicated values of the congestion parameter, $\mathrm{k}$.

\section{Table 1}

Joint expected payoff for each cutoff strategy and alternating

\begin{tabular}{|l|c|}
\hline Strategy & Joint Expected Payoff \\
\hline $\mathrm{C}=0.5$ (always Enter) & 2 \\
$\mathrm{C}=1.5$ & 2.61 \\
$\mathrm{C}^{*}=2.5$ & 2.88 \\
$\mathrm{C}=3.5$ & 2.64 \\
$\mathrm{C}=4.5$ & 1.73 \\
$\mathrm{C}=5.5$ (always Exit) & 0 \\
Alternating & 3 \\
\hline
\end{tabular}

The pair's joint expected payoff for each symmetric pure-strategy cutoff and alternating. 
Table 2

2x2 Payoff Matrix for Cooperation and Coordination Games

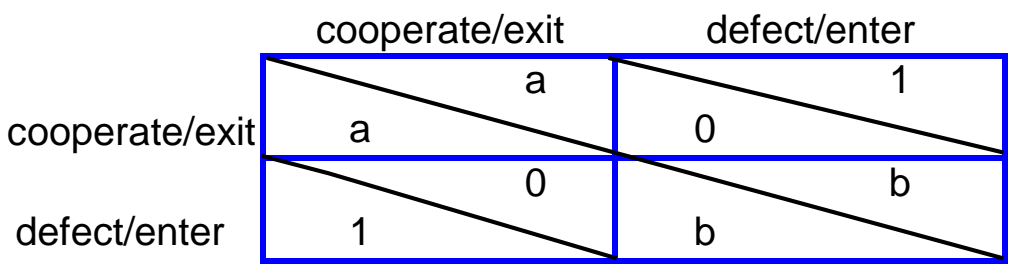
our game
$: 1 / 2>b>a=0$
prisoners' dilemma/public goods
$: a>b>0,1>a>1 / 2$
threshold public goods
$: 1 / 2>a>0>b$
market-entry game
$: a=0>b$
chicken
$: 1>a>0>b$
battle of the sexes
$: a, b>1$

Table 3

Entry by Number and by Treatment

\begin{tabular}{||ccc|}
\hline Number & After & Never \\
\hline 1 & $16.3 \%$ & $30.8 \%$ \\
2 & $29.4 \%$ & $53.8 \%$ \\
3 & $86.2 \%$ & $88.8 \%$ \\
4 & $98.0 \%$ & $95.6 \%$ \\
5 & $98.5 \%$ & $95.4 \%$ \\
\hline \hline Overall & $67.7 \%$ & $74.4 \%$ \\
\hline
\end{tabular}

For each number, each cell indicates the percentage of entry across all subjects in the treatment. 
Table 4 - Random Effects Probit Regressions for Entry Decisions from both treatments

\begin{tabular}{|c|c|c|c|c|}
\hline \multirow[b]{2}{*}{ Variable } & \multicolumn{2}{|c|}{$\overline{(11)}$} & \multicolumn{2}{|c|}{$\overline{(2)}$} \\
\hline & $\begin{array}{l}\text { coefficient } \\
\text { (std. error) }\end{array}$ & $\begin{array}{c}\text { marginal } \\
\text { effect }\end{array}$ & $\begin{array}{l}\text { coefficient } \\
\text { (std. error) }\end{array}$ & $\begin{array}{c}\text { marginal } \\
\text { effect }\end{array}$ \\
\hline Never & $\begin{array}{c}0.174^{\star \star \star} \\
(0.064)\end{array}$ & 0.057 & $\begin{array}{c}0.226^{\star \star \star} \\
(0.071)\end{array}$ & 0.052 \\
\hline $\mathrm{C}_{1.5}$ & --- & --- & $\begin{array}{c}0.617^{\star \star \star} \\
(0.051)\end{array}$ & 0.171 \\
\hline $\mathrm{C}_{2.5}$ & --- & --- & $\begin{array}{c}1.717^{* \star \star} \\
(0.054)\end{array}$ & 0.465 \\
\hline $\mathrm{C}_{3.5}$ & --- & --- & $\begin{array}{c}0.790^{* * *} \\
(0.070)\end{array}$ & 0.172 \\
\hline $\mathrm{C}_{4.5}$ & --- & --- & $\begin{array}{c}0.037 \\
(0.085)\end{array}$ & 0.00 \\
\hline first10 & --- & --- & $\begin{array}{c}-0.144^{\star \star \star} \\
(0.055)\end{array}$ & -0.035 \\
\hline last10 & --- & --- & $\begin{array}{c}0.494^{\star \star \star} \\
(0.060)\end{array}$ & 0.093 \\
\hline constant & $\begin{array}{c}0.534 \\
(0.034)\end{array}$ & & $\begin{array}{l}-1.032 \\
(0.068)\end{array}$ & \\
\hline Number of Obs. & 9920 & & 9920 & \\
\hline$\rho$ & $\begin{array}{c}0.192 \\
(0.024)\end{array}$ & & $\begin{array}{c}0.411 \\
(0.024)\end{array}$ & \\
\hline Log L & -5670 & & -2984 & \\
\hline
\end{tabular}

The dependent variable is subject $i$ 's entry decision in period $t$.
${ }^{* * *} p$-value less than .01
** $p$-value less than .05
* $p$-value less than .10

Random effects Probit regression results from pooled data from both treatments. The entry decision of subject $i$ in period $t$ is regressed on a dummy variable for the Never treatment (regression (1)), interacted with controls for the subject's number and the first and last 10 rounds of play (regression (2)). 
Table 5

Strategy Inference Results by Treatment

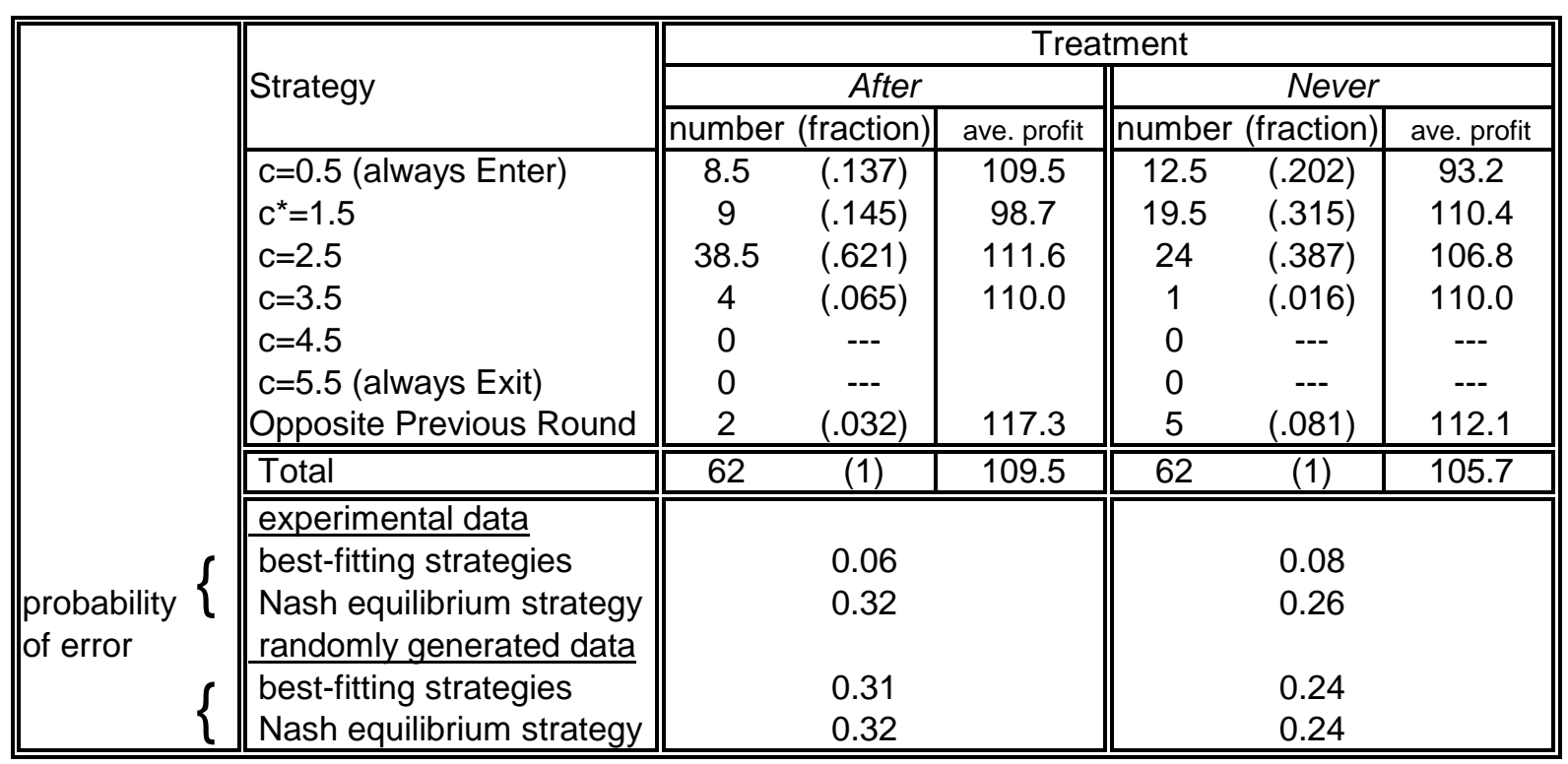

Number (fraction) of subjects whose best-fit strategy based on their decisions from rounds 11-70 corresponds to the one indicated and the average nominal profit earned by subjects playing each strategy type. The average error rates from classifying subjects according to these inferred strategies and from the assumption that all subjects play the Nash equilibrium are shown along with the average error rates for randomly generated data. 
Table 6 - Random Effects Probit Regressions by Treatment

\begin{tabular}{|c|c|c|c|c|c|c|c|c|}
\hline \multirow[b]{4}{*}{ Variable } & \multicolumn{8}{|c|}{ Treatment } \\
\hline & \multicolumn{4}{|c|}{ After } & \multicolumn{4}{|c|}{ Never } \\
\hline & \multicolumn{2}{|c|}{ (1) } & \multicolumn{2}{|c|}{$(2)$} & \multicolumn{2}{|c|}{ (3) } & \multicolumn{2}{|c|}{ (4) } \\
\hline & $\begin{array}{l}\text { coefficient } \\
\text { (std. error) }\end{array}$ & $\begin{array}{c}\text { marginal } \\
\text { effect }\end{array}$ & $\begin{array}{l}\text { coefficient } \\
\text { (std. error) }\end{array}$ & $\begin{array}{c}\text { marginal } \\
\text { effect }\end{array}$ & $\begin{array}{l}\text { coefficient } \\
\text { (std. error) }\end{array}$ & $\begin{array}{c}\text { narginal } \\
\text { effect }\end{array}$ & $\begin{array}{l}\text { coefficient } \\
\text { (std. error) }\end{array}$ & $\begin{array}{c}\text { narginal } \\
\text { effect }\end{array}$ \\
\hline $\mathrm{C}_{1.5}$ & $\begin{array}{c}0.483^{* \star *} \\
(0.081)\end{array}$ & 0.133 & $\begin{array}{c}0.478^{\star * *} \\
(0.081)\end{array}$ & 0.130 & $\begin{array}{c}0.755^{\star \star \star} \\
(0.070)\end{array}$ & 0.243 & $\begin{array}{c}0.780^{\star * *} \\
(0.071)\end{array}$ & 0.251 \\
\hline $\mathrm{C}_{2.5}$ & $\begin{array}{c}2.125^{\star * *} \\
(0.080)\end{array}$ & 0.587 & $\begin{array}{c}2.107^{* * *} \\
(0.081)\end{array}$ & 0.578 & $\begin{array}{l}1.375^{\star * *} \\
(0.078)\end{array}$ & 0.412 & $\begin{array}{c}1.348^{\star * *} \\
(0.079)\end{array}$ & 0.403 \\
\hline $\mathrm{C}_{3.5}$ & $\begin{array}{l}1.018^{\star \star *} \\
(0.109)\end{array}$ & 0.223 & $\begin{array}{l}1.039^{\star * *} \\
(0.110)\end{array}$ & 0.225 & $\begin{array}{c}0.567^{\star \star \star} \\
(0.095)\end{array}$ & 0.149 & $\begin{array}{c}0.579^{\star * \star} \\
(0.096)\end{array}$ & 0.151 \\
\hline $\mathrm{C}_{4.5}$ & $\begin{array}{c}0.225 \\
(0.151)\end{array}$ & 0.000 & $\begin{array}{c}0.215 \\
(0.152) \\
\end{array}$ & 0.000 & $\begin{array}{l}0.0018 \\
(0.107) \\
\end{array}$ & 0.000 & $\begin{array}{l}-0.002 \\
(0.107) \\
\end{array}$ & 0.000 \\
\hline first10 & $\begin{array}{l}-0.219^{\star *} \\
(0.092)\end{array}$ & -0.057 & $\begin{array}{c}-0.204^{\star *} \\
(0.095)\end{array}$ & -0.052 & $\begin{array}{l}-0.005 \\
(0.085)\end{array}$ & 0.000 & $\begin{array}{c}0.056 \\
(0.088)\end{array}$ & 0.000 \\
\hline last10 & $\begin{array}{c}0.543^{* * *} \\
(0.091)\end{array}$ & 0.104 & $\begin{array}{c}0.541^{\star * *} \\
(0.095)\end{array}$ & 0.102 & $\begin{array}{c}0.471^{* * *} \\
(0.084)\end{array}$ & 0.108 & $\begin{array}{c}0.395^{\star * *} \\
(0.088)\end{array}$ & 0.093 \\
\hline ienteredprevious2 & $\begin{array}{l}-0.117^{*} \\
(0.066)\end{array}$ & -0.028 & --- & --- & $\begin{array}{l}-0.004 \\
(0.058)\end{array}$ & 0.000 & --- & --- \\
\hline ienteredprevious2*half1 & -- & --- & $\begin{array}{c}-0.252^{\star \star \star} \\
(0.086)\end{array}$ & -0.064 & --- & --- & $\begin{array}{l}-0.087 \\
(0.075)\end{array}$ & 0.000 \\
\hline ienteredprevious2*half2 & --- & --- & $\begin{array}{c}0.009 \\
(0.086) \\
\end{array}$ & 0.000 & --- & --- & $\begin{array}{c}0.028 \\
(0.078) \\
\end{array}$ & 0.000 \\
\hline iexitedprevious2 & $\begin{array}{c}0.142 \\
(0.103)\end{array}$ & 0.000 & -- & --- & $\begin{array}{c}0.670^{\star * *} \\
(0.123)\end{array}$ & 0.136 & -- & --- \\
\hline iexitedprevious2*half1 & --- & --- & $\begin{array}{l}0.287^{* *} \\
(0.145)\end{array}$ & 0.059 & --- & --- & $\begin{array}{c}0.879^{\star \star \star} \\
(0.188)\end{array}$ & 0.155 \\
\hline iexitedprevious2*half2 & --- & --- & $\begin{array}{c}0.010 \\
(0.141)\end{array}$ & 0.000 & --- & --- & $\begin{array}{c}0.471^{\star * \star} \\
(0.165)\end{array}$ & 0.102 \\
\hline uenteredprevious2 & $\begin{array}{c}0.103 \\
(0.070)\end{array}$ & 0.000 & -- & --- & $\begin{array}{c}0.203^{\star * *} \\
(0.058)\end{array}$ & 0.055 & -- & --- \\
\hline uenteredprevious2*half1 & --- & --- & $\begin{array}{c}0.129 \\
(0.087)\end{array}$ & 0.000 & --- & --- & $\begin{array}{c}0.095 \\
(0.074)\end{array}$ & 0.000 \\
\hline uenteredprevious2*half2 & --- & --- & $\begin{array}{c}0.049 \\
(0.089)\end{array}$ & 0.000 & --- & --- & $\begin{array}{c}0.301^{\star \star \star} \\
(0.078)\end{array}$ & 0.077 \\
\hline uexitedprevious2 & $\begin{array}{l}-0.175^{\star} \\
(0.098)\end{array}$ & -0.044 & --- & --- & $\begin{array}{l}-0.164 \\
(0.106)\end{array}$ & 0.000 & --- & --- \\
\hline uexitedprevious2*half1 & --- & --- & $\begin{array}{c}-0.078 \\
(0.135)\end{array}$ & 0.000 & --- & --- & $\begin{array}{l}-0.256^{*} \\
(0.144)\end{array}$ & -0.076 \\
\hline uexitedprevious2*half2 & --- & --- & $\begin{array}{l}-0.264^{\star *} \\
(0.136)\end{array}$ & -0.069 & --- & --- & $\begin{array}{l}-0.081 \\
(0.151)\end{array}$ & 0.000 \\
\hline constant & $\begin{array}{l}-1.213 \\
-0.105\end{array}$ & & $\begin{array}{l}-1.191 \\
(0.106)\end{array}$ & & $\begin{array}{l}-1.054 \\
(0.069)\end{array}$ & & $\begin{array}{c}-1.05 \\
(0.070)\end{array}$ & \\
\hline Number of Obs. & 4836 & & 4836 & & 4836 & & 4836 & \\
\hline$\rho$ & $\begin{array}{c}0.411 \\
(0.042)\end{array}$ & & $\begin{array}{c}0.419 \\
(0.044)\end{array}$ & & $\begin{array}{c}0.536 \\
(0.028)\end{array}$ & & $\begin{array}{c}0.541 \\
(0.028)\end{array}$ & \\
\hline Log L & -1245.8 & & -1241.0 & & -1560.3 & & -1553.2 & \\
\hline
\end{tabular}

The dependent variable is subject $i$ 's entry decision in period $t$.

*** $p$-value less than $.01{ }^{*} p$-value less than .10

Random-effects Probit regression results by treatment. The entry decision of subject $i$ in period $t$ is regressed on dummy variables for the numbers received, whether the game is in the first 10 or last 10 rounds of play and whether the subject or his opponent made same decision in the previous 2 periods (regressions (1) and (3)), interacted with the first 40 and last 40 rounds of play in regressions (2) and (4). 
Table 7

Comparison of Nominal and Monetary Payoffs in Initial and Follow-Up Treatments

\begin{tabular}{|c|c|c|c|c|c|c|c|}
\hline \multirow{2}{*}{ Number } & \multirow{2}{*}{ Outcome } & \multicolumn{2}{|c|}{ Payoff } & \multirow{2}{*}{ Number } & \multirow{2}{*}{ Outcome } & \multicolumn{2}{|c|}{ Payoff } \\
\hline & & Nominal & Monetary & & & Nominal & Monetary \\
\hline$\overline{---}$ & Exit & 0 & 0 & $\overline{---}$ & Exit & 0 & 0 \\
\hline 1 & Enter Alone & 1 & 0.6 & 101 & Enter Alone & 101 & 1.765 \\
\hline 2 & Enter Alone & 2 & 1.2 & 102 & Enter Alone & 102 & 1.783 \\
\hline 3 & Enter Alone & 3 & 1.8 & 103 & Enter Alone & 103 & 1.8 \\
\hline 4 & Enter Alone & 4 & 2.4 & 104 & Enter Alone & 104 & 1.817 \\
\hline 5 & Enter Alone & 5 & 3 & 105 & Enter Alone & 105 & 1.835 \\
\hline 1 & Both Enter & 0.333 & 0.2 & 101 & Both Enter & 33.667 & 0.588 \\
\hline 2 & Both Enter & 0.667 & 0.4 & 102 & Both Enter & 34 & 0.594 \\
\hline 3 & Both Enter & 1 & 0.6 & 103 & Both Enter & 34.333 & 0.6 \\
\hline 4 & Both Enter & 1.333 & 0.8 & 104 & Both Enter & 34.667 & 0.606 \\
\hline 5 & Both Enter & 1.667 & 1 & 105 & Both Enter & 35 & 0.612 \\
\hline
\end{tabular}

Table 8

Entry by Number and by Treatment

\begin{tabular}{||ccc|}
\hline Number & After100 & Never100 \\
\hline 101 & $62.6 \%$ & $60.0 \%$ \\
102 & $58.9 \%$ & $61.1 \%$ \\
103 & $63.1 \%$ & $67.1 \%$ \\
104 & $65.6 \%$ & $67.2 \%$ \\
105 & $65.7 \%$ & $67.8 \%$ \\
\hline \hline Overall & $63.3 \%$ & $64.8 \%$ \\
\hline
\end{tabular}

For each number, each cell indicates the percentage of entry across all subjects in the treatment. 
Table 9

Strategy Inference Results (Treatments with Constant of 100 Added to Entry Values)

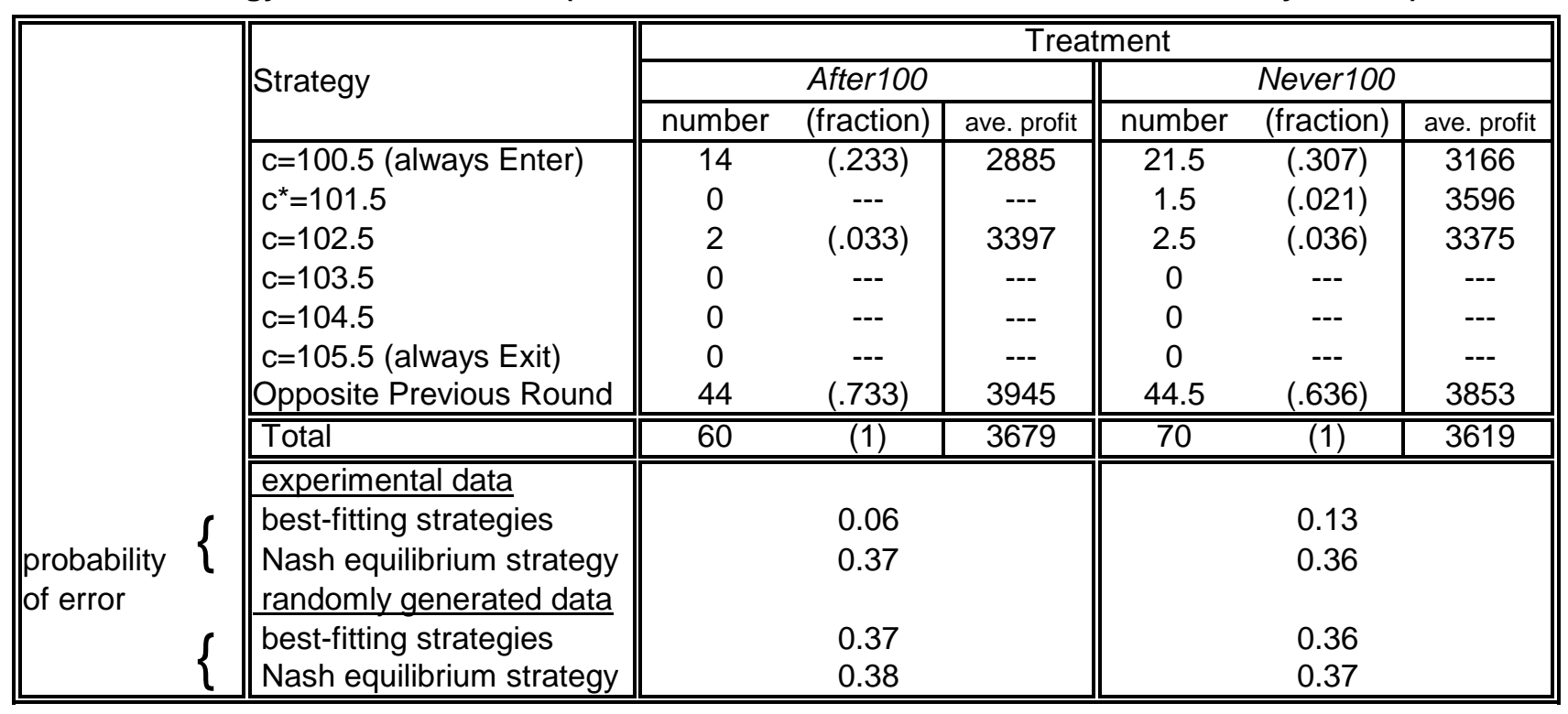

Number (fraction) of subjects whose best-fit strategy based on their decisions from rounds 11-70 corresponds to the one indicated and the average nominal profit earned by subjects playing each strategy type. The average error rates from classifying subjects according to these inferred strategies and from the assumption that all subjects play the Nash equilibrium are shown along with the average error rates for randomly generated data. 
Figure $2 a$ and $\mathbf{2 b}$
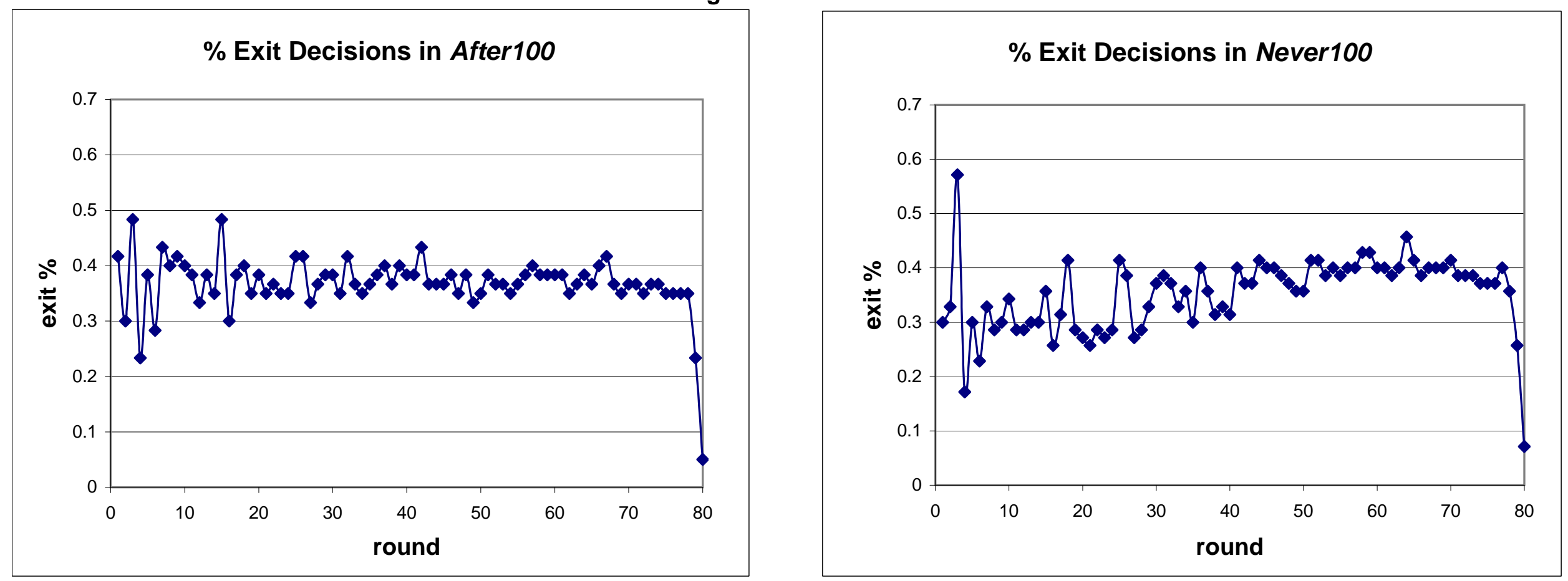

The percentage of exit decisions by round pooled across all subjects for the After100 (left panel) and Never100 (right panel) treatments. 